\title{
Use of rabies immune globulin in seven urban emergency rooms in Pakistan
}

\author{
Naseem Salahuddin ${ }^{\mathrm{a}}$, Khadija. Mubashar ${ }^{\mathrm{b}}$, Naila Baig-Ansari ${ }^{\mathrm{a}}$ \\ ${ }^{a}$ Indus Hospital, Karachi 75190, ${ }^{b}$ Ziauddin Memorial Hospital, Karachi 74700, Pakistan
}

\begin{abstract}
Background: Human rabies is a fatal infectious disease that is entirely preventable if correct and timely postexposure prophylaxis is given. Unfortunately, rabies immune globulin (RIG) administration, a life-saving biological, is often avoided by Emergency Room health care providers (HCPs).

Objective: To understand the practices of HCPs for administration of RIG in severe dog-bite exposures, which are common causes of emergency room visits in Pakistan.

Methods: A cross-sectional study was conducted among 103 HCPs working in seven hospitals in three cities of Pakistan.

Results: Of 103 HCPs who responded to the questionnaires, 97.1\% had administered rabies vaccine and 31.1\% had administered ERIG in the past three years of their practice; three quarters said they would prefer to use HRIG if available; $35 \%$ said they would not inject wounds at all, $24.3 \%$ would only vaccinate a patient with severe dog bites, but not administer RIG. More than 55\% were concerned about the cost of human and equine RIG.

Conclusion: Although there is awareness about use of RIG, this life saving biological is grossly underused because of poor availability in Emergency Rooms (ERs) even though stocked by local dealers. Animal bite centers must make RIG available for severe exposures.
\end{abstract}

Keywords: Animal bites, medical officers' practices, rabies immune globulin, rabies prophylaxis

Rabies is primarily a zoonotic infection caused by a virus that is transmitted through the saliva of a rabid animal, most commonly dogs. It is transmitted to humans by a bite or lick on broken skin or mucous membrane. Death from rabies occurs if prophylaxis is delayed, inadequate, or incomplete. Rabies causes an estimated 55,000 global deaths annually, most of which are in Asia [1].

With an estimated human: dog ratio of 7.4 in urban areas and 14.3 in rural areas of Asia, around 2.5 billion people are at risk of rabies [2]. After a potential rabies threat, it is vital that the patient be given proper postexposure prophylaxis (PEP). This includes thorough wound washing to remove saliva and dirt; administration of a complete course of potent antirabies vaccine (ARV) which stimulates the body's immune system to produce antibodies actively, and local infiltration of rabies immune globulin (RIG) into the wound that neutralizes rabies virus at site before native antibodies from vaccination appear. However, in many developing countries RIG is either under used

Correspondence to: Naseem Salahuddin, Indus Hospital, Karachi 75190, Pakistan.E-mail: naseemsal@hotmail.com or not used at all even in high risk exposures. This study was done to understand the reasons why health care providers in emergency rooms fail to administer RIG where indicated.

\section{Materials and methods}

To investigate attitudes of physicians regarding administration of RIG to rabies-exposed patients, a questionnaire was prepared consisting of 27 questions with options. There were 103 responses from seven institutions in three cities of Pakistan- Islamabad, Lahore, and Karachi. The questionnaires were completed by health care providers based either in ERs or in private clinics where dog bite victims present frequently for management. The Statistical Package for Social Sciences (ver. 16, SPSS, Chicago, IL, USA) was used to analyze the data. Frequencies and percentages were presented for HCP practice location, the number of severe dog bites seen by a facility per year, the treatment usually administered by the HCP for treating severe dog bites, and the concerns of those HCPs why they are not giving RIG. Type of facility was categorized as ER, government sector non-ER, private sector non-ER, whereas the number of severe dog bites seen annually was 
categorized as no animal bite cases, 100 or less, and more than 100 cases of animal bites. For these categorical variables, chi-square test was used to assess statistical significance between with their concerns. A $p<0.05$ was considered significant.

\section{Results}

Of 103 health care providers who responded to the questionnaires, $51.5 \%$ were in an Emergency Room (ER) setting and nearly half had seen more than 100 severe dog bites in a year. Almost all the HCPs interviewed had administered rabies vaccine (97.1\%), 31.1\% had administered ERIG; and 63.1\% had administered tetanus toxoid in the past three years of their practice. Almost three-quarters indicated that they would use HRIG if it were made available to them. Among these HCPs, 35\% said they would never inject wounds, $24.3 \%$ would only vaccinate a patient with severe dog bite, but not administer rabies immunoglobulin (RIG). More than 55\% were concerned about the cost of ERIG (Table 1). The primary concerns of the 70 HCPs who stated they did not give ERIG for severe dog bites were anaphylactic reaction $(57.1 \%)$, cost (52.9\%), and local adverse reactions (45\%), almost 36\% had concerns about local wound infection being made worse by injections followed by $29 \%$ who indicated unfamiliarity of injecting wounds (Table 2). A larger proportion of HCPs from facilities that had never seen severe dog bites were more concerned about their unfamiliarity with injecting wounds (62.5\%), in comparison to those seeing a hundred or less (18\%) and those facilities seeing more than 100 severe dog bite cases per year (31\%) $(p<0.05)$ as shown in Table 3. Statistically significant differences were seen in the proportion of HCPs who were concerned over anaphylactic reaction from ERIG based on their facility setting. Nearly 93\% of the HCPs from private non-ER settings, and 72\% from ER settings were concerned over anaphylactic reaction in comparison to $31 \%$ HCPs from a government non-ER setting $(p<0.001)$ (Table 4).

Table 1. Facility and HCP description

\begin{tabular}{ll}
\hline & $\mathbf{n}(\mathbf{\%})$ \\
\hline Practice Location & \\
Govt. setting-Non ER & $32(31.1)$ \\
Private-non ER & $15(14.6)$ \\
ER setting & $53(51.5)$ \\
Other & $3(2.9)$ \\
HCP Age & \\
20-29 & $42(40.8)$ \\
30-39 & $31(30.1)$ \\
40-49 & $16(15.5)$ \\
50+ & $4(3.9)$ \\
unknown & $10(9.7)$ \\
Severe dog bites seen per year & \\
None & $8(7.8)$ \\
1-100 cases & $51(49.5)$ \\
100+ & $42(40.8)$ \\
Don't Know & $4(1.9)$ \\
Treatment administered by HCP for severe dog bite & \\
Tetanus Toxoid & $65(63.1)$ \\
Rabies Vaccine & $100(97.1)$ \\
Equine RIG & $32(31.1)$ \\
\% of HCPs who would administer HRIG, if available & $74(71.8)$ \\
\% HCPs who would only vaccinate pt with severe dog & $36(35.0)$ \\
\% HCPs concerned about cost of ERIG & \\
& $25(24.3)$ \\
& $57(55.3)$ \\
& \\
\hline
\end{tabular}


Table 2. Concerns of those HCPs not giving ERIG for severe dog bites $(\mathrm{n}=70)$

\begin{tabular}{ll}
\hline Concerns & $\mathbf{n}(\mathbf{\%})$ \\
\hline High cost of ERIG & \\
Not concerned & $32(45.7)$ \\
Concerned & $37(52.9)$ \\
Don’t know & $1(1.4)$ \\
Local adverse reactions & \\
$\quad$ Not concerned & $38(54.3 \%)$ \\
Concerned & $32(45 \%)$ \\
Anaphylaxis & \\
Not concerned & $30(42.9 \%)$ \\
Concerned & $40(57.1 \%)$ \\
Local wound infection made worse by injections & \\
Not concerned & $43(61.4 \%)$ \\
Concerned & $25(35.7 \%)$ \\
Don’t know & $2(2.8 \%)$ \\
Causing additional pain to pt by injecting wounds & \\
Not concerned & $52(74.3 \%)$ \\
Concerned & $18(25.7 \%)$ \\
Unfamiliarity of injecting wounds & \\
Not concerned & $49(70.0 \%)$ \\
Concerned & $20(28.6 \%)$ \\
Don’t know & $1(1.4 \%)$ \\
\hline
\end{tabular}

Table 3. Concerns regarding administering ERIG, based on facility dog-bite burden ( $\mathrm{n}=101)$

\begin{tabular}{|c|c|c|c|c|c|}
\hline Concerns & $\begin{array}{l}\text { No animal bite } \\
\quad(n=8)\end{array}$ & $\begin{array}{l}\text { 1-100 animal bites } \\
\quad(n=51)\end{array}$ & $\begin{array}{l}>100 \text { animal bites } \\
\quad(n=42)\end{array}$ & $\begin{array}{c}\text { Total } \\
(n=101)\end{array}$ & $p$ \\
\hline \multicolumn{6}{|c|}{ Local adverse reactions } \\
\hline Not concerned & $3(37.5 \%)$ & $22(43.1 \%)$ & $22(52.4 \%)$ & $47(46.5 \%)$ & \multirow[t]{2}{*}{ NS } \\
\hline Concerned & $5(62.5 \%)$ & $29(56.9 \%)$ & $20(47.6 \%)$ & $54(53.5 \%)$ & \\
\hline \multicolumn{6}{|l|}{ Anaphylaxis } \\
\hline Not concerned & $3(37.5 \%)$ & $16(31.4 \%)$ & $18(42.9 \%)$ & $37(36.6 \%)$ & \multirow[t]{2}{*}{ NS } \\
\hline Concerned & $5(62.5 \%)$ & $35(68.6 \%)$ & $24(57.1 \%)$ & $63(63.4 \%)$ & \\
\hline \multicolumn{6}{|c|}{ Local wound infection made worse by injections } \\
\hline Not concerned & $5(62.5 \%)$ & $29(59.2 \%)$ & $27(64.3 \%)$ & $61(61.6 \%)$ & \multirow[t]{2}{*}{ NS } \\
\hline Concerned & $3(37.5 \%)$ & $20(40.8 \%)$ & $15(35.7 \%)$ & $38(38.4 \%)$ & \\
\hline \multicolumn{6}{|c|}{ Causing additional pain to pt by injecting wounds } \\
\hline Not concerned & $6(75.0 \%)$ & $39(76.5 \%)$ & $31(73.8 \%)$ & $76(75.2 \%)$ & \multirow[t]{2}{*}{ NS } \\
\hline concerned & $2(25.0 \%)$ & $12(23.5 \%)$ & $11(26.2 \%)$ & $25(24.8 \%)$ & \\
\hline \multicolumn{6}{|c|}{ Unfamiliarity of injecting wounds } \\
\hline Not concerned & $3(37.5 \%)$ & $41(82.0 \%)$ & $29(69.0 \%)$ & $73(73.0 \%)$ & \multirow[t]{2}{*}{0.02} \\
\hline Concerned & $5(62.5 \%)$ & $9(18.0 \%)$ & $13(31.0 \%)$ & $27(27.0 \%)$ & \\
\hline
\end{tabular}


Table 4. Concerns regarding administering ERIG, based on facility type $(\mathrm{n}=103)$

\begin{tabular}{|c|c|c|c|c|c|c|}
\hline Concerns & $\begin{array}{c}\text { Govt. Setting } \\
\text { (non-ER) } \\
(\mathbf{n}=32)\end{array}$ & $\begin{array}{l}\text { Private Setting } \\
\text { (non-ER) } \\
(\mathbf{n}=15)\end{array}$ & $\begin{array}{l}\text { ER Setting } \\
(\mathrm{n}=53)\end{array}$ & $\begin{array}{l}\text { Other } \\
(n=3)\end{array}$ & $\begin{array}{l}\text { Total } \\
(n=103)\end{array}$ & $p$ \\
\hline \multicolumn{7}{|c|}{ Local adverse reactions } \\
\hline Not concerned & $20(62.5 \%)$ & $4(26.7 \%)$ & $22(41.5 \%)$ & $2(66.7 \%)$ & $48(46.6 \%)$ & \multirow[t]{2}{*}{0.08} \\
\hline Concerned & $12(37.5 \%)$ & $11(73.3 \%)$ & $31(58.5 \%)$ & $1(33.3 \%)$ & $55(53.4 \%)$ & \\
\hline \multicolumn{7}{|l|}{ Anaphylaxis } \\
\hline Not concerned & $22(68.8 \%)$ & $1(6.7 \%)$ & $14(26.4 \%)$ & $1(33.3 \%)$ & $37(36.6 \%)$ & \multirow{3}{*}{$<0.001$} \\
\hline Concerned & $10(31.2 \%)$ & $14(93.3 \%)$ & $38(71.7 \%)$ & $2(66.7 \%)$ & $63(63.4 \%)$ & \\
\hline DK & - & - & $1(1.9 \%)$ & - & $1(1.0 \%)$ & \\
\hline \multicolumn{7}{|c|}{ Local wound infection made worse by injections } \\
\hline Not concerned & $23(71.9 \%)$ & $7(46.7 \%)$ & $29(54.7 \%)$ & $2(66.7 \%)$ & $61(61.0 \%)$ & \multirow{3}{*}{ NS } \\
\hline Concerned & $8(25.0 \%)$ & $7(46.7 \%)$ & $23(43.4 \%)$ & $1(33.3 \%)$ & $39(38.4 \%)$ & \\
\hline DK & $1(3.1 \%)$ & $1(6.7 \%)$ & $1(1.9 \%)$ & - & $3(2.9 \%)$ & \\
\hline \multicolumn{7}{|c|}{ Causing additional pain to pt by injecting wounds } \\
\hline Not concerned & $23(71.9 \%)$ & $10(66.7 \%)$ & $41(77.4 \%)$ & $3(100 \%)$ & $77(74.6 \%)$ & \multirow{3}{*}{ NS } \\
\hline Concerned & $8(25.0 \%)$ & $5(33.3 \%)$ & $12(22.6 \%)$ & - & $25(25.3 \%)$ & \\
\hline DK & $1(3.1 \%)$ & - & - & - & $1(1.0 \%)$ & \\
\hline \multicolumn{7}{|c|}{ Unfamiliarity of injecting wounds } \\
\hline Not concerned & $22(68.8 \%)$ & $10(66.7 \%)$ & $39(73.6 \%)$ & $2(66.7 \%)$ & $73(70.9 \%)$ & \multirow{3}{*}{ NS } \\
\hline Concerned & $8(25.0 \%)$ & $5(33.3 \%)$ & $13(24.5 \%)$ & $1(33.3 \%)$ & $27(26.2 \%)$ & \\
\hline DK & $2(6.2 \%)$ & - & $1(1.9 \%)$ & - & $3(2.9 \%)$ & \\
\hline
\end{tabular}

\section{Discussion}

Rabies affects mainly the poor and underprivileged population of developing countries where stray dogs roam the streets [3]. Unimmunized pet dogs are also often responsible for transmitting rabies to humans, especially to children who are unable to escape or defend themselves from attacks. Once the virus enters the body and symptoms begin, the disease is almost always fatal. However, timely and correct postexposure prophylaxis can prevent the disease.

Thorough wound washing can reduce the chances of rabies by 30-40\%. Wound severity must be categorized according to depth, and further management instituted. A superficial scratch may be treated with vaccine alone given according to schedule, while deep or multiple wounds indicate a larger risk and must be treated with passive rabies immune globulin in addition to vaccination. The quantity of RIG must be calculated according to the patient's weight and infiltrated into the wounds to kill the virus and provide immediate protection until antibodies against the virus are mounted through use of the vaccine.

In a previous study, conducted in eight Asian countries including Pakistan [4], and in which the author participated; only 22\% of Category III bite victims received RIG. Several other studies done in Pakistan have revealed serious gaps in understanding of wound severity classification and correct use of vaccine and RIG [4, 6, 7].

Three classes of RIG are presently available: human (HRIG), equine (ERIG), and highly purified F (ab)2' ERIG products. HRIG is produced from donors under strict manufacturing conditions and is devoid of adverse effects [8]. However, its production is limited and its cost is exorbitant and hence unaffordable in developing countries. ERIG is produced from horse serum and in the past was responsible for up to $40 \%$ adverse effects and even anaphylaxis. Current ERIG products are highly purified and almost devoid of adverse effects. WHO no longer recommends skin tests, but exercises caution in their use [8]. F (ab)2' products of ERIG are cleared more rapidly than ordinary ERIG and have virtually no side effects. However, experience has caused many end users nevertheless to be apprehensive of using the product. Many potentially rabid bites are still inadequately treated, resulting in many rabies deaths.

Production of the conventional polyclonal RIGs is labor intensive and requires a modern horse farm for success. This results in global shortages, though poor distribution is also a serious problem. A positive 
breakthrough in recent years is the innovation of a human monoclonal antibody (mAb), which is likely to replace RIG $[9,10]$. mAb would be produced in larger quantities and would in due course, overcome RIG scarcity and expense. Phase 4 trials are currently underway. Whether these problems will be more affordable than HRIG in the poorer countries remains to be seen.

This study clearly shows that, although many HCPs in the study appreciate the importance of using RIG, they have not acquired sufficient upgraded information on RIG quality improvement. ERIG and purified $\mathrm{F}(\mathrm{ab}) 2^{\prime}$ ERIG are safe and effective and carry very low adverse reaction rates similar to those of most injected antibiotics, including penicillins. National Regulatory Authorities should facilitate import and distribution of ERIG, and all Rabies Prevention Centers should be supplied ERIG at affordable cost. Moreover, nurses and doctors should receive training on correct use of vaccine and RIG infiltration.

The authors have no conflict of interest to report.

\section{References}

1. Wilde H, Khawplod P, Khamoltham T, Hemachudha T, Tepsumethanon V, Lumlerdacha B, et al. Rabies control in South and Southeast Asia. Vaccine. 2005; 23:2284-9.

2. Knobel DL, Cleaveland S, Coleman PG, Fevre EM, Meltzer MI, Miranda ME, et al. Re-evaluating the burden of rabies in Africa and Asia. Bull WHO. 2005;
83:360-8.

3. Burney MI, Khan ZH, Lari FA. The rabies problem in Pakistan. Trop Doct. 1976; 6:60-2.

4. Dodet B, Goswami A, Gunasekera A, de Guzman F, Jamali S, Montalban C, et al. Rabies awareness in eight Asian countries. Vaccine. 2008; 26:6344-8.

5. Parviz S, Chotani R, McCormick J, Fisher-Hoch S, Luby S. Rabies deaths in Pakistan: results of ineffective post-exposure treatment. Int J Infect Dis. 2004; 8: 346-52.

6. Parviz S, Luby S, Wilde H. Postexposure treatment of rabies in Pakistan. Clin Infect Dis. 1998; 27:751-6

7. Salahuddin N, Jamali S, Ibraheem K, Sardar S. Awareness about rabies post exposure prophylaxis in Pakistan among patients and health care workers: results from an Asian rabies expert bureau study. J Coll Physicians Surg Pak; 21:491-4.

8. WHO International Expert Consultation on rabies; WHO Geneva. 2012.

9. Champion JM, Kean RB, Rupprecht CE, Notkins AL, Koprowski H, Dietzschold B, et al. The development of monoclonal human rabies virus-neutralizing antibodies as a substitute for pooled human immune globulin in the prophylactic treatment of rabies virus exposure. J Immun Methods. 2000; 235:81-90.

10. Nagarajan T, Rupprecht CE, Dessain SK, Rangarajan PN, Thiagarajan D, Srinivasan VA. Human monoclonal antibody and vaccine approaches to prevent human rabies. Curr Top Microbiol Immunol. 2008; 317:67-101. 